\title{
The Positive Impact on Emergency Department Length of Stay of a Clinical Practice Guideline-Directed Process for Direct Admission from the Emergency Department Computed Tomography Scan Suite to the Intensive Care Unit of Highest Level Trauma Activation Patients
}

${ }^{1}$ Ehyal Shweiki, ${ }^{2}$ Kathleen D Martin, ${ }^{3}$ Anne Judge, ${ }^{4}$ Megan Asher

\begin{abstract}
Introduction: Patients presenting to the emergency department (ED) as the highest level trauma activation (T1) are characteristically severely injured, having met physiologic or anatomic derangement criteria as defined by the American College of Surgeons' (ACS) committee on trauma (COT) guidelines for field triage. ED overcapacity is a significant issue for the vast majority of hospitals in the United States. Multiple published reports show that ED overcapacity leads to delays in patient care and increased morbidity and mortality. Such negative impacts include those on trauma patients' specifically. Based on literature-reported improved outcomes for decreased ED length of stay (LOS) in critically ill patients, we aimed for the expeditious transport from the ED computed tomography (CT) scanner suite to the intensive care unit (ICU), of T1 patients who were ultimately deemed to need ICU level of care. More, we hypothesized that a well-delineated, well-integrated multidisciplinary "direct to ICU" clinical practice guideline (CPG) would enable our stated aim to be achieved in a consistent manner.
\end{abstract}

Methods: This was a retrospective evaluation of patients admitted to a Level II trauma center over a 12 month period. The cohorts were patients who were highest level (T1) and whose immediate post-ED destination was the ICU. ED LOS of stay (LOS) was assessed for both pre-CPG (January-April, 2016) and post-CPG (May to December 2016) groups. Patients going directly to the operating room $(\mathrm{OR})$, interventional radiology (IR), ED deaths or medical-surgical unit (MedSurg), i.e., ward/ floor admissions were excluded.

Results: The average ED LOS preimplementation of the CPG was 159 minutes ( $S D \pm 8.8$ ). The post-CPG implementation ED LOS average was 49 minutes (SD \pm 8.87 ). Thus the ED LOS was 110 minutes less, post-CPG implementation. As a noteworthy comparative benchmark, the 2016 "one-way-street" article from the Massachusetts General Hospital reported an 82 minute median ED LOS for trauma patients admitted directly from the ED CT to the ICU.

\footnotetext{
${ }^{1}$ Surgeon, ${ }^{2}$ Nurse, ${ }^{3,4}$ Nurse Practitioner

1-4Trauma Program, Lankenau Medical Center, Wynnewood, Pennsylvannia, USA
}

Corresponding Author: Ehyal Shweiki, Surgeon,Trauma Program, Lankenau Medical Center, Wynnewood, Pennsylvannia, USA, Phone: 4845720171, Email: shweikie@mlhs.org
Conclusion: Implementation of a multidisciplinary "direct to ICU" CPG enabled the very expeditious, reproducible and process-oriented transport of critically ill trauma patients from the ED CT suite to the ICU. In support of contemporary, evidence-based efforts to enhance trauma patient outcomes while also addressing ED overcapacity concerns, We propose this "direct to ICU" CPG model for use by other trauma centers.

Keywords: ED-LOS, ED overcrowding, Direct from ED to ICU, ICU, Trauma.

How to cite this article: Shweiki Ehyal, Martin KD, Judge A, Asher M. The Positive Impact on Emergency Department Length of Stay of a Nothing to do-Directed Process for Direct Admission from the ED Computed Tomography Scan Suite, to the Intensive Care Unit, of Highest Level Trauma Activation Patients. Panam J Trauma Crit Care Emerg Surg 2018;7(2):89-96.

Source of support: Nil

Conflict of interest: None

\section{INTRODUCTION}

Patients presenting to the ED of trauma centers as the highest level of T1, are characteristically severely injured, having met either physiologic or anatomic derangement criteria as defined by the ACS COT guidelines for field triage. $^{1}$

Since the first reports in the late 1980s, the issue of ED overcapacity has been a substantial issue for the vast majority of hospitals in the United States. Highlighting this was a 2002 survey by the American Hospital Association noting that "the percentage of large hospital EDs that are consistently operating at or above capacity has reached 90\%." 2 The issue received national regulatory attention the following year when the general accounting office (GAO) reported its findings directly to the United States Senate Finance Committee, regarding "ED crowding" 3 As an even more stark expression of a national and now "epidemic (healthcare) concern," in June 2006, the Institute of Medicine published the landmark study, "Hospital-based emergency care: At the breaking point." ${ }^{4}$ (Flow Chart 1) 
Flow Chart 1: Trauma program clinical practice guideline

Subject: Trauma "code activation" transition of care from ED CT scan suite to the ICU procedure:

Communication between providers enables best transitions of care when a patient is transferred from one location to another. Timely and effective communication is paramount in promoting patient safety and care.

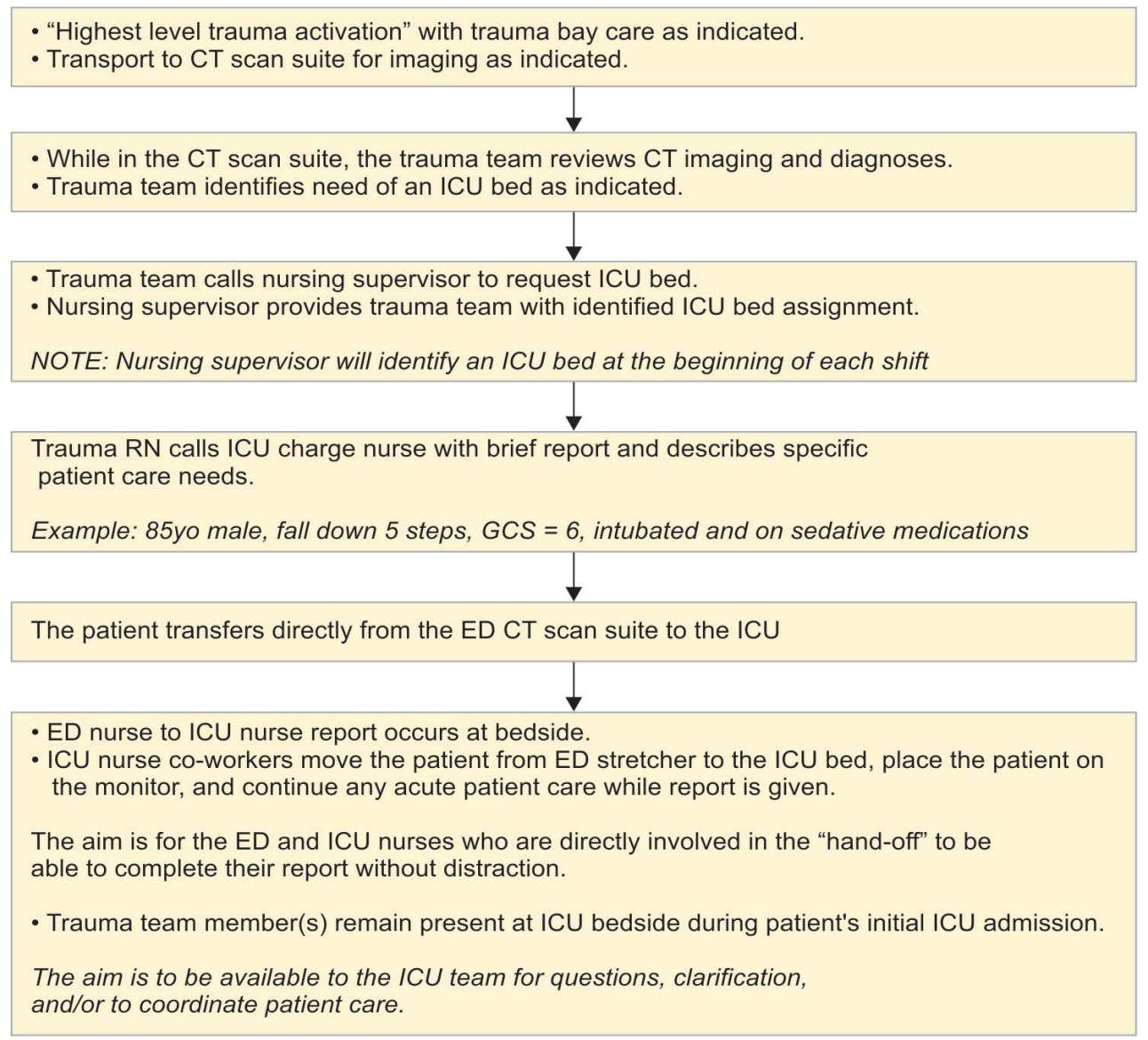

Responsibilities of the Trauma Team during ED-ICU "Handoff"

- State name of patient, DOB, and attending surgeon

- Identification of the specific members of the ICU accepting team (RN, RT, PCT...)

- State reasons for patient's ICU admission/mechanism of injury

- List and summarize patient injuries/problems

- List relevant events that have occurred prior to ICU admission (i.e., pre-hospital; ED course including vs, blood/fluids/vasopressor administration; relevant radiologic studies performed; if intubated note the reason; if analgesics/sedatives/paralytics given then clarify doses)

- List past medical, surgical, social, and ROS as available

- List home medications and allergies

- State prior contact with, or availability of family members or surrogates

- Describe invasive lines, drains, chest tubes, foley, etc. and any specific management instructions

- List Services consulted and their pertinent recommendations as completed

- List plan of care for next 24 hours (e.g., surgeries, studies, items that need follow-up, etc.)

- Verbalize special instructions (i.e., non-weight bearing, spinal immobilization, etc.)

- Obtain urine drug screen (UDS) and ETOH level if not obtained in the ED

Though the causes of ED overcrowding are multifactorial, the dominant reported factor relates to patient flow within the hospital, specifically the increased needs of higher acuity patients who present to EDs, with these patients' longer dispositions into resource-limited ICU beds. $^{3-7}$
In sum, ED overcrowding and longer ED LOS is a well-documented issue in American healthcare. Importantly, subsequent to the above reports, multiple publications have shown that longer ED LOS is associated with increased morbidity and mortality, particularly when the ED LOS relates to critically ill patients. 
Reported metrics comparing outcomes of patients for varied ED LOS include hospital LOS, increased resource use during hospitalization, poorer functional outcome at discharge after stroke, risk of pneumonia in intubated patients, as well as hospital mortality. ${ }^{8-16}$ With documentation of increased morbidity and mortality from increased ED LOS, efforts to reduce ED LOS have ensued, and several specific approaches have been published (Table 1). ${ }^{17-21}$

Regarding the critically ill trauma patient, the need for safe, expeditious transfer of patients out of the ED, into the ICU applies as well. Yet appropriate time spent in the ED involves stabilization of airway, breathing and circulation (ABC) with initial resuscitation, completing advanced trauma life support (ATLS) delineated primary and secondary surveys, as well as triage-directed diagnostic imaging. The latter often involves multiple advanced imaging techniques including computed tomography CT scans. In all, appropriate ED care for critically ill trauma patients involves a balance between time necessarily spent in ED processes of care, and a needed expeditious transfer out of the ED into ICU.

Contemporary literature finds that for high acuity patients, i.e. those requiring ICU admission after the ED phase of care, expeditious transfer from the ED to the ICU will have a positive impact on outcomes. In advancing this need to decrease ED LOS for critically ill patients, several institutions have published reports on novel interventions whereby ED patients were expedited in transfer from the ED to a respective ICU (both medical and surgical ICU patient admissions). ${ }^{17-21}$ With these reported varied efforts, significant improvements in average ED LOS were shown. ${ }^{9,16-21}$

Importantly, an expedited transfer of patients from the ED to the ICU involves several resources and logistical issues including a readily and continually available ICU bed; a readily and continually available hospital "bed coordinator" to facilitate admission; as well as a readily and continually available ICU nurse to receive a "handoff" report.

Based on literature-reported concerns of increased ED LOS, and with the numerous evidence-based publications reporting improved outcomes with decreased ED LOS in critically ill patients, we aimed to implement a process of direct, expedited transfer of T1 patients from the ED CT scanner to the ICU. Specifically, our intervention implemented a CPG directed process. We hypothesized that a well-delineated, well integrated multidisciplinary, "direct to ICU" CPG would enable our stated aim to be achieved in a consistent manner. Operationally, our CPG included: (a) installation of a dedicated phone in the ED CT suite from which to call the nursing supervisor/bed coordinator; (b) an "open/held ICU bed" at all times, for T1 patients; (c) an ICU nurse available at all times to respond to the $\mathrm{ED}$ for $\mathrm{T} 1$ activations and to continue to provide care if ICU admission indicated; (d) a planned ED nurse as well as the trauma advanced practitioner bedside "handoff" report to be given in the ICU, to the ICU nurse team.

\section{METHODS}

This retrospective cohort study was conducted at a 350 bed acute care hospital that is accredited by the Pennsylvania

Table 1: Literature review of process improvements for patients admitted from the ED to the ICU

\begin{tabular}{|c|c|c|c|c|}
\hline Study & Unit & Process & $\begin{array}{l}\text { ED LOS } \\
\text { preintervention mean (min)* }\end{array}$ & $\begin{array}{l}\text { Decreased ED LOS } \\
\text { postintervention mean (min)* }\end{array}$ \\
\hline Bhakta et al. & SICU & 24/7 “open" ICU bed & $252 \pm 240$ & $186 \pm 126$ \\
\hline Fuentes et al. & SICU & Direct from CT scan to ICU & $\begin{array}{l}\text { Median } 331 \\
\text { (range } 229-474 \text { ) } \\
\text { *only median reported }\end{array}$ & $\begin{array}{l}\text { Median } 82 \text { (range } 65-116 \text { ) } \\
\text { *only median reported }\end{array}$ \\
\hline Ko et al. & SICU & $\begin{array}{l}\text { ICU bed requested prior to } \\
\text { transport to CT scan }\end{array}$ & $179 \pm 124$ & $143 \pm 97$ \\
\hline \multirow[t]{2}{*}{ Elliott et al. } & MICU & $\begin{array}{l}\text { Patients requiring } \mathrm{MICU} \\
\text { admission managed by MICU } \\
\text { RN, PA, and MD. }\end{array}$ & 530 & 374 \\
\hline & & $\begin{array}{l}\text { "MICU Alert Team" development } \\
\text { and implementation. }\end{array}$ & & \\
\hline McCoy et al. & MICU & $\begin{array}{l}\text { Eliminated MICU resident } \\
\text { screening. MICU fellow and } \\
\text { attending evaluate the patient. } \\
\text { Resident orders written within one } \\
\text { hour in the MICU. }\end{array}$ & 375 & 324 \\
\hline Shweiki et al. & SICU & $\begin{array}{l}\text { Direct from CT scan suite to ICU CPG, } \\
\text { including "open" ICU bed } 24 / 7\end{array}$ & $159 \min$ & 49 \\
\hline
\end{tabular}


Trauma Systems Foundation (PTSF) as a Level II trauma center. Data were queried from the Collector ${ }^{\circledR}$ trauma registry for all admission to the trauma service from January to December, 2016. We included all admissions with a primary diagnosis of trauma-based upon ICD-10-CM injury codes S00-S99, T07-T79/ICD-9-CM injury codes 800-995) excluding both ICD-10-CM T15-T19.9/930-939.9 and CD-10-CM S72.00-S72.26/ICD-9-CM codes 820.00820.9. Patients who died in the ED, or who went directly to the OR or interventional radiology, and those patients admitted to a non-ICU Med Surg unit were excluded. The cohort consisted of patients who presented as the highest levels of activation (T1) and whose immediate post ED destination was the ICU. The "direct from ED CT to ICU CPG" was implemented in May 2016 (Fig. 1).

Note the CPG involved the following providers: ED nurse, trauma service attending and advanced practitioner, nursing supervisor/bed coordinator, ICU "charge" and bedside nurse. All personnel were available 24/7. An available "open ICU" bed was ensured $24 / 7$, and would be preferentially located in the surgical ICU (SICU), though a "back-up" bed was available in the medical ICU (MICU), in the event the SICU was not accessible.

(Note, on occasions when admission of trauma patients to the MICU occurred, the nursing staff involved in the care of the patient were personnel who had completed specialty nurse training in trauma care).

Documented times were available for review of both patient arrivals in the ED as well as actual patient departure time from the ED. ED LOS in minutes was assessed for both pre-CPG implementation (January to April 2016) and post-CPG implementation (May to December 2016).

This study did not meet need of Institutional Review Board review given the retrospective cohort via. trauma registry data set metrics, and the ability to de-identify individual patients without compromising the research intent.

\section{RESULTS}

The mean age in years for the pre-CPG group was $54( \pm 32)$ versus a post-CPG group which was $52( \pm 28)$. The mean Injury Severity Score (ISS) for the pre-CPG group was $12.7( \pm 10.7)$ versus $13.4( \pm 13.9)$ for the post CPG group. Neither age nor ISS were found to be statistically significant differences between the two groups.

The average ED LOS in minutes pre-CPG was 159 $(\mathrm{SD} \pm 8.8)$ versus post-CPG $49(\mathrm{SD} \pm 8.87)$. This was a statistically significant difference, $\mathrm{P}<0.0001$ (Table 2). Hospital LOS in days was $2.6( \pm 2.1)$ in the pre-CPG group and $3.1( \pm 2.5)$ in the post CPG group. Ventilator days pre-CPG were $0.6(+0.8)$, and $1.4(+1.3)$ post-CPG. Mortality was $29 \%$ pre-CPG and $10 \%$ post-CPG. There were no statistically significant differences in these metrics between the pre- and post-CPG groups. A t-test was used for analysis of the data utilizing a comparison of means which calculated the difference between the observed means of two independent samples. The means were taken as significantly different if the $\mathrm{p}<0.0001$.

Table 2: ED length of stay, age and ISS-pre and post CPG implementation

\begin{tabular}{llll}
\hline Mean $( \pm S D)$ & $\begin{array}{l}\text { Pre-CPG } \\
\text { Jan-Apr 2016 }\end{array}$ & $\begin{array}{l}\text { Post-CPG } \\
\text { May-Dec 2016 }\end{array}$ & $\begin{array}{l}\text { Significance } \\
\text { level }\end{array}$ \\
\hline $\begin{array}{l}\text { ED LOS in } \\
\text { minutes }\end{array}$ & $159(\mathrm{SD} \pm 8.8)$ & $49(\mathrm{SD} \pm 8.8)$ & $\mathrm{p}<0.001$ \\
Age mean & $54( \pm 32)$ & $52( \pm 28)$ & $\mathrm{p}=0.9143$ \\
ISS mean & $12.7( \pm 10.7)$ & $13.4( \pm 13.9)$ & $\mathrm{p}=0.9125$ \\
\hline
\end{tabular}

\section{DISCUSSION}

In an effort to improve patient outcomes multiple prior publications have reported the need to reduce ED LOS, particularly for the critically ill. Studies to date have addressed patient cohorts across both the MICU and SICU. With reported efforts to date, significant reductions in ED LOS have been effected without negative impact on mortality. Also as reported to date, impacts on morbidity have been favorable, though more detailed research needed to enable more declarative assessments regarding improved patient care outcomes. ${ }^{2-17}$ It is noteworthy that the publications referenced cross a patient spectrum from medically ill to surgically ill, and more specifically, include trauma patients. Thus in an effort to further improve patient outcomes, continued institutional efforts addressing ED LOS in critically ill individuals is needed, with the subsequent application, nationally, of those processes deemed successful. More, we posit that with large amounts of trauma-related data being increasingly available via the National Trauma Data Bank, the ACS trauma quality improvement project (TQIP) and/or PTSF registries, research on outcomes related to ED LOS for $\mathrm{T} 1$ would be very informative and potentially drive a new metric of best practice in trauma care. Currently, no such standardized nor best practice metric exists. As ours is a retrospective review strong conclusions are not possible. This not with Standing we posit our data are significant and should serve as a targeted metric for best practice relating to ED LOS in this patient cohort. Future research, done on a larger scale would further assess our hypothesis.

Our efforts focused on a multidisciplinary collaboration between the trauma program, ED, ICU, nursing staff, and radiology department. This resulted in a CPG that expedited admission to the ICU, from the ED, of highest level trauma activation patients. The CPG streamlined 
the process from initial assessment, through patient stabilization in the trauma bay, to CT scan, and then to direct ICU admission. Our CPG significantly decreased ED LOS, and without a negative impact on patient morbidity or mortality. Preparation and implementation of this CPG-directed process required a concerted approach by multiple individuals across departments. Of note, a multidisciplinary approach enabling safe and expeditious transfer of patients across hospital geography has been described in varied formats to date. Examples include use of a "MICU alert team (MAT)," use of a direct ED Attending to ICU Attending triage communication model, use of an "24/7 open trauma bed protocol in the surgical ICU," as well as direct transfer of patients from the ED CT scan suite to the ICU. ${ }^{17-21}$ We incorporated into our CPG, components of these varied reported multidisciplinary processes. Importantly, we specifically ensured that leadership personnel from the departments stated were involved in the development of the CPG. Further, during the preparation and implementation phases of the CPG, it was continually reviewed at our monthly trauma administrative committee (TAC) as well as our monthly Trauma Program Multidisciplinary Peer Review Committee meetings. We strongly believe that having multidisciplinary leadership involvement at these meetings facilitated "buy-in" as well as the subsequent education of clinical personnel who would be implementing the CPG.

From an operational perspective an important factor enabling our expeditious decision-making for need of an ICU bed relates to the attending trauma surgeon's presence in the CT scan suite at the time of imaging. Real-time review of $\mathrm{CT}$ imaging with resultant interpretation thus occurs. If questions arise regarding CT interpretation, a radiologist is available $24 / 7$ for discussion and clarification. Hence, critical diagnoses are made essentially at the time of CT imaging, thus facilitating immediate and appropriate disposition (i.e., ICU, MedSurg floor, return to ED for other care/disposition, etc).

Regarding continual access to an "open ICU" bed, our institution has a detailed process for ensuring continued access to ICU levels of care. In brief, trauma patients have priority access to the SICU, with "overflow" access to the MICU as needed.

Note, Our CPG includes "staying one ICU bed ahead."

Namely, a room and enough staff are ensured to be available at all times in the event of need. Addressing the staffing issue, the ICU "charge" nurse will assume a patient care assignment, as needed, while any "on-call" nursing personnel are accessed for further support.

Overflow of SICU nontrauma patients occurs in the MICU, the "step-down" unit, or the post-operative care units (all of which have ICU capabilities). Our institution also has a dedicated/distinct cardiothoracic $I C U$.

With the above described institutional ICU level of care access, we encountered no episode whereby a trauma service requested ICU bed was not available, nor were we made aware of the negative impact of our CPG process on access for other patients to needed ICU beds. Corroborating this assessment is that the trauma program medical director (and lead author ES) attends the institution's Critical Care Workgroup Committee where such metrics are reviewed.

In sum, we found that the development and use of a well-delineated, well-integrated, multidisciplinary CPG-directed process enabled a marked improvement in ED LOS compared to both our prior ED LOS, as well as compared to other published reports. ${ }^{17-21}$ In fact our study showed an average ED LOS of 49 minutes. This compares very favorably to the two other published reports on the streamlined admission of critically ill trauma patients from the ED CT suite to the ICU-a mean of 144 minutes as reported by Ko et al.; and a median of 82 minutes as reported by Fuentes et al. ${ }^{20}$ Importantly the range in the latter paper was 65-116 minutes, the lower end still above the 49 minute average shown as possible in our study.

Potential limitations of this cohort study are noted. Firstly, the sample size is small. We do not consider this to be a major limitation for several reasons: (a) multiple published reports across varied institutions have established the concern of ED overcrowding as leading to increased ED LOS and negative impacts on morbidity and mortality. Hence our small cohort does not impact on these prior reported/evidence-based findings; (b) Our process of expedited transfer of critically ill trauma patients from ED to ICU is not a novel idea, having been effected and published by larger cohorts. ${ }^{20,21}$ Rather, our specific aim was to develop and implement a well-delineated, well-integrated and multidisciplinary process, i.e., a CPG, to effect even further improvements in prior reported advances in ED LOS. Of note, Ko et al. In their paper, "decreased transport time to the surgical ICU" hypothesized that "additional human factors interventions emphasizing improved communication and coordination can further reduce time spent in the ED." ${ }^{21}$ It is these specific "human factors interventions" that we sought to address via. our CPG. Hence it is meaningful, that developing and implementing such a human factor-related process in care, i.e., a CPG brought such a considerable further reduction in ED LOS as compared to prior published reports. ${ }^{17-21}$ This is a retrospective report. However, given the evidence-based reports to date of the positive impact on morbidity/mortality of decreasing ED LOS in critically ill patients, we believe we are beyond clinical equipoise to safely consider a prospective 
randomized trial. Still, as noted, further research across much larger cohorts (e.g., ACS TQIP, PTSF) could elucidate/validate our findings.

An additional potential limitation of our paper relates to the limited period of time over which the study was conducted. However, given that a specific intent of the CPG was to serve as a well-delineated process in care, we believe the time period was sufficient to assess this purpose. Importantly, to ensure durability of our results we reviewed our CPG process related to $\mathrm{T} 1$ patients admitted directly to the ICU for the subsequent twelve months, January-December 2017. Despite our average ISS increasing to 17.68 , our meantime-from arrival of T1 patients, thru CT scan to ICU, was 54 minutes. This closely mirrored our prior results. Thus we believe our CPG shows, at least early sustainability. Nevertheless, it will be important to continue to track our ED LOS related to $\mathrm{T} 1$ patients needing ICU care, to ensure our CPG-directed process remains effective, and that our ED LOS for this cohort does not drift off our current metric.

\section{CONCLUSION}

ED overcrowding in the United States is an established concern, and the resultant increased ED LOS for critically ill patients specifically has been reported as a negative impact on morbidity and mortality. Our implementation of a well-delineated, well-integrated, multidisciplinary "direct to ICU" CPG enabled a very expeditious, reproducible and process-oriented mechanism for transport of critically ill trauma patients from the EDCT scan suite to the ICU. Of note, our mean ED LOS for this patient cohort (i.e., highest level trauma activation critically ill trauma patients) was $49( \pm 8)$ minutes, which is well in advance of prior published reporting on expedited ED LOS for critically ill patients.

In support of contemporary, evidenced-based efforts to enhance trauma patient outcomes, while also addressing ED overcapacity concerns, we propose this "direct to ICU CPG" model for use by other trauma centers.

The authors would like to acknowledge the contributions of nina betts, trauma registrar, Rikki Teja, trauma registrar, and Darlene Gondell, trauma PI coordinator for their contribution to data validation, analysis and mutual teamwork. Also, they are thankful to Fran Cusick, Director of Nursing for her support in facilitating the implementation of this process.

\section{REFERENCES}

1. Resources for Optimal Care of the Injury Patient 2014. 6th ed. Chicago,IL: Committee on Trauma, American College of Surgeons; 2014.

2. The Lewin Group: Emergency Department Overload: A Growing Crisis. The Results of the American Hospital
Association Survey of Emergency Department (ED) and Hospital Capacity. Falls Church, VA: American Hospital Association; 2002. Available from: http://www.hospitalconnect.com/AHQA/press_room-info/content/EdoCrisisSlides. pdf(Accessed 9th September 2017).

3. General Accounting Office. Hospital Emergency Departments, Crowded Conditions Vary Among Hospitals and Communities. Washington, D.C.: United States General Accounting Office; 2003. Available from: https://www.gao. gov/new.items/d03460.pdf(Accessed 7th September 2017).

4. Institute of Medicine.Hospital-Based Emergency Care: At the Breaking Point. Washington, D.C.: The National Academies Press; 2007.

5. Cowan R., Trzeciak S. Clinical Review: Emergency Department Overcrowding and the Potential Impact on the Critically Ill.Critical Care. 2004;9:291-295.

6. Olshaker J. Managing Emergency Department Overcrowding. Emergency Medicine Clinics of North America. 2009;27:593-603.

7. Eitel D., Rudkin S., Malvehy M., Killeen J., Pines J. Improving Service Quality By Understanding Emergency Department Flow: A White Paper and Position Statement Prepared for the American Academy of Emergency Medicine.Journal of Emergency Medicine. 2010;38:70-79.

8. Carr B., Kaye A., Wiebe D., Garcias V., Schwab C., Reilly P. Emergency Department Length of Stay: A Major Risk Factor for Pneumonia in Intubated Blunt Trauma Patients. Journal of Trauma. 2007; 63:9-12.

9. Richardson J., Franklin G., Santos A., Harbrecht B., Danzi D., Coleman R., et al. Effective Triage Can Ameliorate the Deleterious Effects of Delayed Transfer of Trauma Patients From the Emergency Department to the ICU. Journal of the American College of Surgeons. 2009; 208(5): 671-678.

10. Carter A., Pilcher D., Bailey M., Cameron P., Duke G., Cooper J. Is ED Length of Stay Before ICU Admission Related to Patient Mortality?.Emergency Medicine Australasia. 2010; 22(2): 145-150.

11. Rincon F., Mayer S., Rivolta J., Stillman J., Boden-Albala B., Elkind M., et al. Impact of Delayed Transfer of Critically Ill Stroke Patients from the Emergency Department to the Neuro-ICU. Neurocritical Care. 2010; 13(1): 75-81.

12. Singer A., Thode Jr H., Viccellio P., Pines J. The Association Between Length of Emergency Department Boarding and Mortality. Academic Emergency Medicine. 2011; 18(12), 13241329.

13. Flabouris A., Jeyadoss J., Field J., Soulsby T. Association Between Emergency Department Length of Stay and Outcome of Patients Admitted Either to a Ward, Intensive Care or High Dependency Unit. Journal of the Australasian Academy of Critical Care Medicine. 2012; 14: 191-197.

14. Hung S., Kung C., Hung C., Liu B., Liu J., Chew G., et al. Determining Delayed Admission To The Intensive Care Unit For Mechanically Ventilated Patients In The Emergency Department. Critical Care. 2014; 18(4): 485-494.

15. Molina J., Seow E., Heng B., Chong W., Ho B. Outcomes of Direct and Indirect Medical Intensive Care Unit Admissions From the Emergency Department of an Acute Care Hospital: A Retrospective Cohort Study. BMJ Open. 2014; 4(11): p.e005553.

16. Elliott D., Williams K., Wu P., Kher H., Michalec B., Reinbold N., et al. An Interdepartmental Care Model to Expedite Admission From the Emergency Department to the Medical 
ICU. The Joint Commission Journal on Quality and Patient Safety. 2015; 41(12):542-549.

17. Siletz A., Jin K., Cohen M., Lewis C., Tillou A., Cryer H., et al. Emergency Department Length of Stay in Critical Nonoperative Trauma. Journal of Surgical Research. 2017; 214: 102-108.

18. Bhakta A., Bloom M., Warren H., Shah N., Casas T.,Ewing, T., et al. The Impact of Implementing a 24/7 Open Trauma Bed Protocol in the Surgical Intensive Care Unit on Throughput and Outcomes. The Journal of Trauma and Acute Care Surgery. 2013; 75(1): 97-101.

19. McCoy J., Gale A., Sunderram J., Ohman-Strickland P., Eisenstein, R. Reduced Hospital Duration of Stay Asso- ciated with Revised Emergency Department-Intensive Care Unit Admission Policy: A before and After Study. The Journal of Emergency Medicine. 2015; 49(6): 893900.

20. Fuentes E., Shields J., Chirumamilla N., Martinez M., Kaafarani H., Yeh D., et al.“One-Way-Street” Streamlined Admission of Critically Ill Trauma Patients Reduces Emergency Department Length of Stay. Internal and Emergency Medicine. 2016; 12(7):1019-1024.

21. Ko A., Harada M., Dhillon N., Patel K., Kirillova L., KolusR., et al. Decreased Transport Time to the Surgical Intensive Care Unit. International Journal of Surgery. 2017; 42: 54-57. 


\section{INVITED COMMENTARY}

"The positive impact on ED LOS of a clinical practice guideline-directed process for direct admission from the ED CT scan suite, to the ICU, of highest level trauma activation patients "

The present article describes a successful implementation of a practice guideline, streaming the process of ICU admissions directly for the emergency room. This is a very important issue that can avoid overcrowding of emergency rooms, and expedite the treatment of critically ill patients. Early triage of the sickest patients can improve their outcomes.

In some areas of the world the implementation of these protocols is feasible, in some regions with less resources without intensive care, or intensivist to see over these patients the protocol might not be reproducible. Or at least not with the current system. ${ }^{1,2}$

The burden of critical illness is especially high in countries with limited resources, over $90 \%$ of trauma deaths occur in low or middle income regions. However these regions with the highest acuity also have the largest gaps. ${ }^{3}$

This article highlights the importance that health care systems prioritize the early recognition, triage and care of the critically ill. Producing this type of literature can help build the foundation to restructure the resource allocation in places with the highest need. ${ }^{4,5}$

I congratulate the authors on an excellent paper, and encourage them to work with the Pan-American trauma Society in developing international reproducible protocols to help patients in areas when the need is the greatest.

\section{References}

1. Dunser MW, Towey RM, Amito J, Mer M. Intensive care medicine in rural sub-Saharan Africa. Anaesthesia. 2017;72(2):181-189.

2. Firth P, Ttendo S. Intensive care in low-income countries--a critical need. The New England journal of medicine. 2012;367(21):1974-1976.

3. Mock C, Nguyen S, Quansah R, Arreola-Risa C, Viradia R, Joshipura M. Evaluation of Trauma Care capabilities in four countries using the WHO-IATSIC Guidelines for Essential Trauma Care. World journal of surgery. 2006;30(6):946-956.

4. Derman RJ, Jaeger FJ. Overcoming challenges to dissemination and implementation of research findings in under-resourced countries. Reproductive health. 2018;15(Suppl 1):86.

5. Vukoja M, Riviello E, Gavrilovic S, Adhikari NK, Kashyap R, Bhagwanjee S, et al. A survey on critical care resources and practices in low- and middle-income countries. Global heart. 2014;9(3):337-42 e1-e5. 